\title{
The small impact of p-hacking marginally significant results on the meta-analytic estimation of effect size
}

\author{
Juan Botella ${ }^{1, *}$, Manuel Suero ${ }^{1}$, Juan I. Durán², and Desirée Blázquez \\ 1 Universidad Autónoma de Madrid (Spain). \\ 2 Universidad a Distancia de Madrid (Spain). \\ 3 Universidad de Murcia (Spain).
}

\begin{abstract}
Título: El pequeño impacto del "haqueo" de resultados marginalmente significativos sobre la estimación meta-analítica del tamaño del efecto.

Resumen: La etiqueta $p$-backing $(p H)$ se refiere a un conjunto de prácticas oportunistas destinadas a hacer que sean significativos algunos valores $p$ que deberían ser no significativos. Algunos han argumentado que debemos prevenir y luchar contra el $p H$ por varias razones, especialmente debido a sus posibles efectos nocivos en la evaluación de los resultados de la investigación primaria y su síntesis meta-analítica. Nos focalizamos aquí en el efecto de un tipo específico de $p H$, centrado en estudios marginalmente significativos, en la estimación combinada del tamaño del efecto en el meta-análisis. Queremos saber cuánto deberíamos preocuparnos por su efecto de sesgo al evaluar los resultados de un meta-análisis. Hemos calculado el sesgo en una variedad de situaciones que parecen realistas en términos de prevalencia y de la definición operativa del $p H$. Los resultados muestran que en la mayoría de las situaciones analizadas el sesgo es inferior a una centésima $( \pm 0.01)$, en términos de $d$ o $r$. Para alcanzar un nivel de sesgo de cinco centésimas $( \pm 0.05)$, tendría que haber una presencia masiva de este tipo de $p H$, lo que parece poco realista. Hay muchas buenas razones para luchar contra el $p H$, pero nuestra conclusión principal es que entre esas razones no se incluye que tenga un gran impacto en la estimación metaanalítica del tamaño del efecto.
\end{abstract}

Palabras clave: p-hacking; tamaño del efecto; meta-análisis.

\section{Introduction}

In the past decade there has been much discussion about socalled questionable research practices ( $Q R P s)$, a set of behaviors of scientists that distort the research process and bias the results (Bakker et al., 2012; DeCoster et al., 2015; Earp \& Trafimow, 2015; Pashler \& Harris, 2012). Many claim that QRPs are a major threat to the validity of the conclusions of research. Its consequences occur both when conducting primary research and when doing meta-analytical reviews.

Several expressions have been used to refer to the $Q R P s$, but recently the term $p$-hacking $(p H)$ has been used to describe a set of problematic practices that can lead to systematic bias in conclusions based on published research (Simonsohn et al., 2014a). One result of $p H$ is to select those statistical analyses that lead to non-significant $p$-values being transferred to the region of statistical significance. In practical terms, a result that should have an associated $p$-value greater than the level of significance $(\alpha)$ ends up having a $p$ value below that threshold.

Beyond fraudulent behaviors, such as intentionally fabri-

\footnotetext{
* Correspondence address [Dirección para correspondencia]:

Juan Botella. Universidad Autónoma de Madrid, Facultad de Psicología, Campus de Cantoblanco, c/ Ivan Pavlov, 6, 28049 Madrid (Spain).

E-mail: juan.botella@uam.es

(Article received: 18-06-2020; revised: 23-07-2020; accepted: 31-08-2020)
}

\begin{abstract}
The label $p$-backing $(p H)$ refers to a set of opportunistic practices aimed at making statistically significant $p$ values that should be nonsignificant. Some have argued that we should prevent and fight $p H$ for several reasons, especially because of its potential harmful effects on the assessment of both primary research results and their meta-analytical synthesis. We focus here on the effect of a specific type of $p H$, focused on marginally significant studies, on the combined estimation of effect size in meta-analysis. We want to know how much we should be concerned with its biasing effect when assessing the results of a meta-analysis. We have calculated the bias in a range of situations that seem realistic in terms of the prevalence and the operational definition of $p H$. The results show that in most of the situations analyzed the bias is less than one hundredth $( \pm$ 0.01 ), in terms of $d$ or $r$. To reach a level of bias of five-hundredths ( \pm 0.05 ), there would have to be a massive presence of this type of $p H$, which seems rather unrealistic. We must continue to fight $p H$ for many good reasons, but our main conclusion is that among them is not that it has a big impact on the meta-analytical estimation of effect size.

Keywords: p-hacking; effect size; meta-analysis.
\end{abstract}

cating or falsifying data (Stricker \& Günther, 2019), QRPs are psychologically more "tolerable". They consist of means to "cook" the data by transforming them in various ways, analyzing them with several alternative statistical techniques, analyzing multiple indicators without informing readers of failed methods, performing non-programmed intermediate statistical analyses, selectively eliminating participants, etc. (Fanelli, 2009; Hall \& Martin, 2019; John et al., 2012). If instead of reporting the result of the "normal" analyses, these practices are carried out with the aim of finding a $p$-value below $\alpha$, but without informing readers of the steps taken to produce such results, these procedures inevitably produce two consequences. First, the false positives rate is inflated beyond its nominal value (Bakker et al., 2012; Ioannidis \& Trikalinos, 2007; van Assen et al., 2015), and second, in global terms the results finally reported overestimate the parametric effect size (ES) (Kraemer et al., 1998; Lane \& Dunlap, 1978).

We know that different types of $Q R P^{\prime}$ s can produce somewhat different biases (e.g., Ulrich \& Miller, 2015). We are going to focus on those forms of $Q R P$ in which, in view of a marginally significant result, the researcher does not stop the analysis, but performs actions aimed at obtaining a significant result. Typically, increase the sample and reanalyze (Botella et al., 2006), or analyze sequentially with alternative statistical techniques or transformations.

The use of $p H$ has received considerable attention in re- 
cent years, in the context of the so-called crisis of confidence in psychology (Baker, 2016; Earp \& Trafimow, 2015; Pashler \& Harris, 2012; Pashler \& Wagenmakers, 2012; Yong, 2012). Of course, the focus has been on the biasing effects of $p H$ on the conclusions of single studies. However, a logical extension of those worries is the concern about the potential effects on the conclusions of the meta-analyses carried out in research fields where there is a detectable $p H$ activity. The $p H$ activity in the primary studies could have a kind of domino effect on the meta-analyses that synthesize those studies.

We must prevent and fight QRPs for several good reasons, which we will discuss later. These arguments refer above all to the harmful effects that the results of primary studies contaminated by QRPs can have on their own as well as on the advancement of a scientific field such as psychology. However, we will argue that $p H$ (at least the type of $p H$ we are focusing on here) does not have an important impact on the combined estimate of ES calculated in meta-analyses. In fact, its quantitative impact on meta-analytical estimates is rather small. Our goal in this article is to support the conclusion that calculating the impact of a specific type of $p H$ over a range of meta-analytical conditions can be demonstrated to be surprisingly small to the point of insignificance. To advance the main argument, the reason why the impact of this type of $p H$ on the meta-analysis is small is twofold: (a) the probability that a given study has been subjected to $p H$ such that its results are consequently modified, is relatively small, and (b) the impact of the distortion produced by those few studies in the overall estimation of the population ES is also correspondingly small.

\section{Assessing the prevalence of $p H$}

There are two main sources of evidence for the existence of $p H$ : surveys of researcher practices and statistical analyses of the empirical distributions of ES estimates. Fanelli (2009) performed a meta-analysis of 18 surveys of researchers about their own behavior regarding $Q R P s$, and about those of close colleagues. The results showed that QRPs are present in all scientific branches, with different degrees of prevalence. Unfortunately, the responses also indicate that a non-negligible number of researchers do not perceive some of these QRPs as particularly dishonest. The conclusion is clear: QRPs exist and their presence is not negligible (see, for example, the figure 1 of Brodeur, Lé, Sangnier \& Zylberberg, 2016; or those of Krawczyk, 2015). However, the prevalence estimates vary widely, depending on the method used and the field and subfield analyzed (Banks et al., 2016; Head et al., 2015).

Several statistical procedures have been proposed to test for the presence of $p H$ in a given empirical distribution of $p$ values. On the one hand, the transfer of $p$-values between regions should be visible in the form of an over-abundance of studies in the region of significant values adjacent to nonsignificant values (for example, .03 - .05). Similarly, in the area of $p$-values on the other side of the threshold (just above $\alpha$; for example, .05 - .07) there must be an equivalent short- age of studies. On the other hand, we should notice an overabundance within the area of significant $p$-values adjacent to the threshold with respect to other more remote areas, according to the $p$-curve model fitted. These trends are represented in Figure 1, which shows the smoothed difference between the expected frequency of $p$-values within the range 0 $<p \leq .10$ in the absence of $p H$ and with a transfer of $p$ values from the range $.05<p \leq .10$ to the range $.025<p \leq$ .05 . There is an abrupt step in the threshold value of statistical significance (typically, .05). Depending on the size of the step, the practical effect of $p H$ on the combined estimate of $E S$ will be greater or lesser.

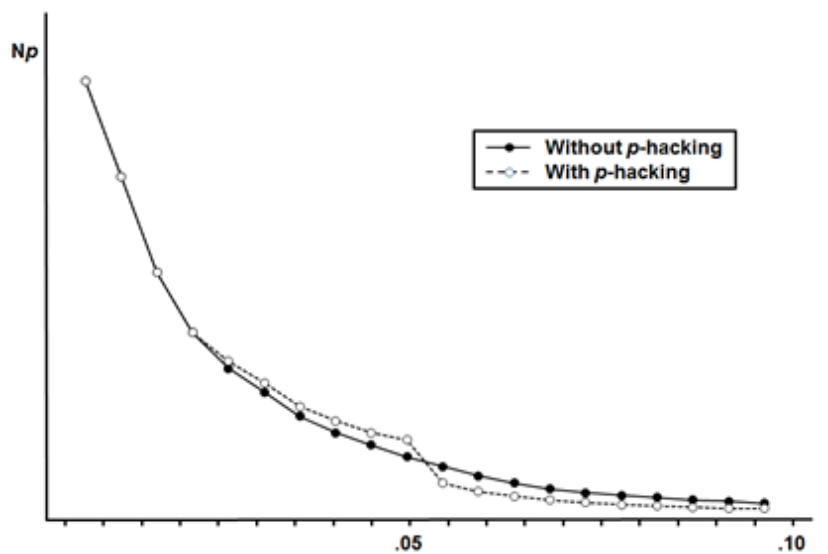

Figure 1. Expected distribution of $p$-values with and without the effect of $p H$.

The effects of $p H$ are hardly detectable in empirical distributions of $p$-values, since the effects of publication bias (PB) mask them (Francis, 2012). The $P B$ against non-significant studies is another threat that biases the combined estimates of the population ES. It is a real and important threat to the validity of conclusions based on meta-analyses that has been well studied and documented for decades (Mueller, 2018; Rothstein et al., 2005; Schneck, 2018; van Aert et al., 2019). Graphically, $P B$ produces a step in the expected distribution of $p$-values that can be very similar to that produced by $p H$. A practical difficulty in assessing the effects of $p H$ with real databases is that, in the scientific fields in which we detect the presence of $p H$, a significant presence of $P B$ is also often detected (e.g., Head et al., 2015; Krawczyk, 2015). Both factors, $p H$ and $P B$, can be reflected in abrupt changes in the distribution of $p$-values around the threshold (Figure 1), between those categorized as significant and those that are not (typically $\alpha=.05$ ), so that their effects are easily confused (Leggett et al., 2013). Consequently, in the calculations that follow, we assume analytic scenarios in which there is $p H$ but no $P B$. In this way, we can assess the pure effect of $p H$. Even so, we will also see the effect of $p H$ when meta-analytic methods are applied only to significant studies, as a way to protect against $P B$. We will see how large the effect of $p H$ is on the estimation of $E S$ when a method such as p-curve analysis is applied to the set of significant studies (Simonsohn et al., 2014b). 
Several studies have assessed the presence of $p H$ in the literature. Head et al. (2015) used data mining techniques to extract distributions of $p$-values from a variety of scientific fields, and they concluded that $p H$ effects are pervasive (but see Hartgerink, 2017). Brodeur et al. (2016) estimated that in the field of economics there is a transfer of between $10 \%$ and $20 \%$ studies from the area of marginally significant to that of significant.

The analysis of the $p$-values reported in more than five thousand articles in experimental psychology journals reported by Krawczyk (2015) reveals several interesting features. Of course, there are anomalies in the empirical $p$ distribution. However, along with an over-abundance of values that are significant but close to the limit of that region (for example, below .05 but close to it), there is also an even greater over-abundance of values above but also very close to the threshold of significance (for example, above .05 but close to it). The interpretation of this pattern is not clear. However, since these anomalies could be due to several factors, especially $P B$, they seem to indicate that $p H$ as such should have had a rather small incidence in the observed distribution of $p$-values (see Krawczyk, 2015, Figures 3 and 4).

Bishop and Thompson (2016) have studied the presence of anomalies in $p$-value distributions through simulations and a re-analysis of the data by Head et al. (2015). They conclude that neither the absence of a bump in the $p$-curve is indicative that there is no $p H$, nor is its presence undoubtedly associated with a distribution contaminated by $p$-values from a nongenuine origin. To reach a sound conclusion, you must assume a specific QRP. In their work they focus on what they call ghost variables, which translate into the parallel analysis of various outcome variables, reporting only the one that is most favorable to the researcher's hypothesis. This form of $Q R P$ is very difficult to detect.

Although these studies provide evidence of the presence of $p H$, and some even estimate its prevalence, most of them do not calculate its quantitative impact on the meta-analytical combined estimate of the ES. They only assess distribution anomalies by testing the null hypothesis of non- $p H$. Simonsohn, Nelson and Simmons (2014a) concluded that it is very unlikely that in a wide range of credible conditions $p H$ is able to yield a non-existing effect. We believe that we must go beyond knowing how probable it is that $p H$ turns a null effect into an incorrect, non-null effect. We want to calculate the impact of a specific type of $p H$ on the combined estimation of ES to know how much we should worry about its biasing effects when assessing the results of a meta-analysis. Specifically, the type of $p H$ that consists of selecting those statistical analyses that lead to $p$-values in the region sometimes referred to as "marginally significant" being transferred to the region of statistical significance ${ }^{1}$. As we have already

1 Our operational definition is associated to the .05 value as the threshold for significance, since it is the most typical alpha level in psychology. In disciplines like pharmacology the typical level of significance is smaller (.01 or even less), while in others like marketing and other branches of economics and sociology it is not uncommon for alpha to be set at .10. suggested, our hypothesis is that although we must protect our research and actively fight $p H$ for several reasons, the estimated effect of this specific type on the combined estimation of the ES in meta-analysis is very limited.

\section{Method}

In what follows we have limited ourselves for simplicity of exposure to one-sided tests with $\alpha=.05$ and a normally distributed test statistic. All arguments generalize to two-sided tests and to other values of $\alpha$. Likewise, we have assumed that all studies have the same sample size. Of course, in real research, the sample sizes vary among the studies, but by assuming equal sizes we simplify the calculations without affecting the conclusions. To calculate the effect of $p H$ on the $E S$ estimation we used a linear combination of the expected values of the test statistic, conditionalized on specific ranges of values obtained in the statistical test. For example, if the statistic tested under the null hypothesis follows a normal distribution, we can obtain its expected value conditionalized on being less than 1.645 (not significant; $p>.05$ ) and on being equal to or greater than 1.645 (significant; $p \leq .05$ ). If we also know the probabilities that the values fall within each of these regions, the total expected value can be obtained by weighing the partial expected values with their corresponding probabilities. The expected conditionalized values are easily obtained from the truncated normal distribution (Johnson, Kotz, \& Balakrishnan, 1994, chap. 13). Specifically, the expected value of the test statistic, $T$, is,

$E(T)=P(T<1.645) \cdot E(T \mid T<1.645)+P(T \geq 1.645) \cdot E(T \mid T \geq 1.645)$

Let's see a simplified, non-realistic example of how we are going to do the calculations, with only two regions, although in the real calculations later we will define a more complex and realistic scenario. If, for example, we randomly transfer studies (with a probability of .50 that a study is transferred) from the region with $p>.05$ to the region with $p$ $\leq .05$, the expected value after these transfers is obtained by recalculating the weights (probabilities) of the conditional expected values. In order to assess the effects of $p H$ in terms of transfers of observations between regions (Figure 2), we will also make two instrumental assumptions. The first states that the probability of transferring a specific study from one region to another is uniform within the region of origin. That is, the probability of transferring, through $Q R P s$, an observation with a $p$-value of (say) between .05 and .10 , is the same for all studies performed while $p$ is within that range.

The second instrumental assumption states that when transferring studies with $.05<p \leq .10$ to the region with $.025<p \leq .05$, the expected value of $T$ is approximately the same as that for studies with genuine $p$-values in that region. These two instrumental assumptions are set to facilitate calculations. Moderate deviations from them do not generate large differences in the results, whereas other approaches require rather arbitrary assumptions. 
In Figure 2 we see that the values that define several regions in the right tail of the distribution of the test statistic under the null hypothesis also define different regions in the true distribution, which is that to the right (with $\delta>0$ ). If we operationally define $p H$ as a transfer (represented by the arrow) of cases from the "marginally significant" region to the "significant" region closest to the significance threshold, we can calculate the expected value of the test statistic and, therefore, the bias produced by a $p H$ of such magnitude. Specifically, we have assumed that there is a probability, $q$, that a value in the region defined as $.05<p \leq .10$ (that is, $1.280 \leq T<1.645)$ is transferred to the region $.025<p \leq .05$ (that is, $1.645 \leq T<1.96$ ). We have calculated the effect of these transfers with conditional probabilities of up to $q=$ .50. This implies a maximum in which half of the results with marginally significant $p$-values end up being significant after being submitted to QRPs. The percentage of exploratory studies submitted to QRPs may be higher, but only a part (up to $50 \%$ of those judged to be marginally significant in our calculations) end up being reported with a statistically significant $p$-value. We believe that even this estimate is high and that in real contexts it is probably smaller than this maximum (Banks et al., 2016; Fanelli, 2009; Fiedler, Schwarz, 2016; John et al., 2012; Martinson et al., 2005). For example, in their analysis of research published in the field of economics Brodeur et al. (2016) estimate that those transfers represent between $10 \%$ and $20 \%$ of marginally significant studies. A survey by Martinson, Anderson and De Vires (2005) found that the self-reported frequencies of incurring QRPs are typically less than $15 \%$. In other analyses, it is assumed that $100 \%$ of the nonsignificant studies are subjected to $p H$ (Simonsohn et al., 2014b), a prevalence that seems exaggerated $^{2}$.

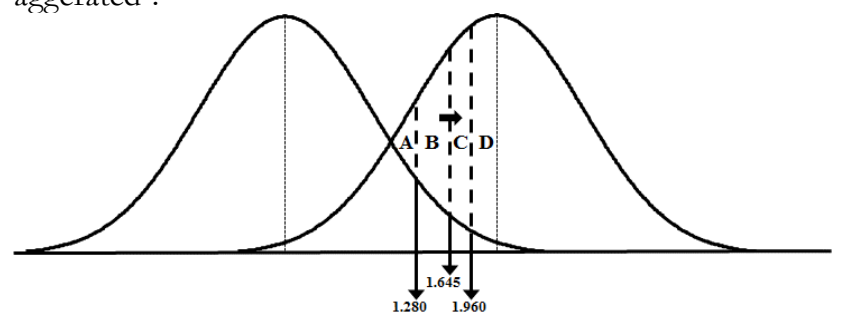

Figure 2. Regions for the statistical test value under the null (left curve) and under the true value (right curve) hypotheses. The letters represent the four regions of the true distribution: A (not significant), B (marginally significant), C (significant with high $p$ ) and D (significant with low $p$ ). The arrow represents the transfer of studies with marginally significant $p$ values to the region with significantly associated $p$ values but close to the threshold defined by $\alpha$.

We have worked with Cohen's $d$ as our effect size index, with two sample sizes that reflect a typically small size in psychology (two groups of 15) and a typically moderate size (two groups of 30) (Marszalek et al., 2011; Rubio-Aparicio et

2 If $100 \%$ of the researchers were active practitioners of the $Q R P s$, our discipline would have reached a level of corruption that would advise forgetting preventive measures and instead close the business and start from the scratch. al., 2018). With larger samples, the effects of $p H$ tend to be even smaller.

The $\delta$ values selected (besides 0 for non-effect) are 0.2 , 0.5 and 0.8 , generally used since Cohen (1988) to designate small, moderate and large standardized mean differences (see also Rubio-Aparicio et al., 2018).

Let us see with a detailed example how we have performed our calculations in the condition with $\delta=0.80$ and $N=15$ (see in Appendix S1, supplemental material, the $R$ syntax that allows to reproduce the calculations reported for this and all other conditions). The expected conditional values have been obtained through the truncated normal distribution. The probability that a study yields a result that is neither significant nor marginally significant $(T<1.28$; region A in Figure 2) is .1904, and the expected value of $T$ for that set of studies is 0.708 . The probability and the expected value of significant studies with $p \leq .025$ (region $\mathrm{D}$ in figure 2) are .5879 and 2.879 , respectively. These two sets of studies are those not affected by the transfer represented by the arrow in Figure 2. The probability that in the test of the null hypothesis, the $T$ value is in the region of "marginally significant" $(1.280 \leq T<1.645$; region B in Figure 2$)$ is equal to .1093 , and its expected conditional value is 1.47 . Finally, the probability that the $p$-value remains in the region of significant values with high $p$-values $(.025<p \leq .05$; region $\mathrm{C}$ in Figure 2) is equal to .1124 and the conditional expected value of $T$ is 1.806 . The total expected value of $T$ in the absence of $p H$ would be:

$E(T)=.1904 \cdot 0.708+.1093 \cdot 1.47+.1124 \cdot 1.806+.5879 \cdot 2.879=2.1911$

Of course, the expected value of $d$ is $\mathrm{E}(d)=\mathrm{E}(T) \cdot$ sqrt $(2 / N)=2.1911 \cdot \operatorname{sqrt}(2 / 15)=0.800$. Let us now see what happens in this body of research if the magnitude of $p H$ is set to $50 \%$ of the marginally significant studies $(q=.5)$, becoming significant with high $p$-values (between .025 and .05). Half of the studies within that range of $p$-values $(.1093 / 2=$ .0546) are transferred to the adjacent area (region $C)$. The expected value of $T$ with that level of $p H$ is:

$E(T)=.1904 \cdot 0.708+.0546 \cdot 1.47+(.1124+.0546) \cdot 1.806+.5879 \cdot 2.879=2.2093$

Then, $\mathrm{E}(d)=2.2093 \cdot \operatorname{sqrt}(2 / 15)=0.807$. The magnitude of the bias produced by this degree of $p H$ in this analytic scenario is $0.807-0.800=0.007$.

\section{Results and discussion}

Table 1 shows the main results in terms of the expected values of $d$ for the different conditions. In each case, the bias is the difference between the value within the table and the true value of $\delta$. In all conditions the bias is less than one hundredth: $|\mathrm{E}(d)-\delta|<0.01$. The greatest biases occur with the smallest sample size $(\mathrm{N}=15)$. The magnitude of the bias effect is generally small and, in many conditions, negligible (less than \pm 0.005 ). We can state that $p H$ is not 
likely to have a relevant quantitative impact on the estimation of $E S$, at least for the conditions studied here. It is easy to understand the two reasons why the impact is small. On the one hand, the probability that a study is subject to $Q R P s$, and the results transferred from the region of marginally significant (region B) to a significant one with high $p$-values (region C, $p$-values between .025 - .05), is small. On the other hand, the conditional expected values of the test statistic in those two adjacent regions are very close. Let us return to the example with $\delta=0.80$ and $\mathrm{N}=15$. The probability that a value of $T$ falls in the region of "marginally significant" $(1.280 \leq \mathrm{T}<1.645)$ equals .1093 , so the maximum transfer we have contemplated $(q=.50)$ would eventually be, in the long run, only $5.46 \%$ of the studies carried out. These transferred studies would have a mean value of $T=1.47(d=$ $0.54)$ in the long run before being subjected to $p H$, while after that process their average value would be $T=1.81(d=$ 0.66). However, the vast majority of studies (in the long run $100-5.45=94.54 \%$ ) would not change their associated values of $T$ (and $d$ ).

Table 1. Expected values of $d$ for the studied conditions, according to the parametric values $(\delta)$, the sample sizes $(N)$, and the probabilities of transferring a "marginally significant" study to a significant one $(q)$.

\begin{tabular}{|c|c|c|c|c|c|c|c|c|}
\hline \multirow[b]{2}{*}{$q$} & \multicolumn{2}{|c|}{$\delta=0$} & \multicolumn{2}{|c|}{$\delta=0.20$} & \multicolumn{2}{|c|}{$\delta=0.50$} & \multicolumn{2}{|c|}{$\delta=0.80$} \\
\hline & $N=15$ & $N=30$ & $N=15$ & $N=30$ & $N=15$ & $N=30$ & $N=15$ & $N=30$ \\
\hline .00 & 0 & 0 & 0.200 & 0.200 & 0.500 & 0.500 & 0.800 & 0.800 \\
\hline .05 & 0 & 0 & 0.201 & 0.200 & 0.501 & 0.501 & 0.801 & 0.800 \\
\hline .10 & 0.001 & 0.000 & 0.201 & 0.201 & 0.502 & 0.501 & 0.801 & 0.800 \\
\hline .15 & 0.001 & 0.001 & 0.202 & 0.201 & 0.503 & 0.502 & 0.802 & 0.800 \\
\hline .20 & 0.001 & 0.001 & 0.202 & 0.202 & 0.503 & 0.502 & 0.803 & 0.801 \\
\hline .25 & 0.002 & 0.001 & 0.203 & 0.202 & 0.504 & 0.503 & 0.803 & 0.801 \\
\hline .30 & 0.002 & 0.001 & 0.204 & 0.203 & 0.505 & 0.503 & 0.804 & 0.801 \\
\hline .35 & 0.002 & 0.002 & 0.204 & 0.203 & 0.506 & 0.504 & 0.805 & 0.801 \\
\hline .40 & 0.003 & 0.002 & 0.205 & 0.204 & 0.507 & 0.504 & 0.805 & 0.801 \\
\hline .45 & 0.003 & 0.002 & 0.205 & 0.204 & 0.508 & 0.505 & 0.806 & 0.802 \\
\hline .50 & 0.003 & 0.002 & 0.206 & 0.205 & 0.509 & 0.506 & 0.807 & 0.802 \\
\hline
\end{tabular}

\section{Generalizing to other conditions}

We want to know whether our conclusion is valid only for the conditions studied until here, and whether it will change significantly in other analytic scenarios. We review the main factors that could affect the conclusions and discuss their potential effects.

(a) Values of $\delta$. The $E S$ values chosen for $\delta$ are those that Cohen proposed as typically small, medium and large ESs. They cover a wide range of representative values of the effects studied by psychologists (Bosco et al., 2015; Richard et al., 2003; Rubio-Aparicio et al., 2018). As the bias reduces from when $\delta$ equals 0.50 to 0.80 , the tendency is that with values greater than 0.80 the bias will be even lower.

(b) Sample size. Sample size does not seem to be a relevant factor, since with $N=15$ and $N=30$ the results are very similar. With $N>30$ the results will be very stable and will show even smaller biases. It is possible that with groups smaller than 15 there is a greater difference, but these sample sizes are not very frequent in psychology, and when they are used, the data are often analyzed with non-parametric techniques. Even so, we have made the calculations with $\delta=$ 0.50 and two groups of $N=10$. The results are essentially the same, reaching one hundredth of bias only when $q=.50$.

(c) The effect size index. Our calculations refer to $\delta$, the standardized mean difference. We ask ourselves whether the conclusions are generalizable to other ES indices. We have made the calculations assuming that the test statistic is normally distributed, so they should be similar for other statistics that have approximate normal distributions. To calculate the bias with other indices, we simply use the corresponding formulas. For example, for Pearson's correlation we have used the values suggested by Cohen for a small, medium and large correlation (rho $=.10, .30$, and .50; see also Richard, Bond, \& Stokes-Zoota, 2003) transformed to Fisher's $Z$ and with samples of size 15 and 30 . The results appear in Table S1 (tables S1 to S6 are included in the supplemental material). In all conditions the bias is less than one hundredth, $|\mathrm{E}(r)-\varrho|<0.01$. In the majority they are less than half a hundredth, although to reach that value the percentage of transfers must reach $50 \%$ and the sample must be small $(\mathrm{N}$ $=15)$. Therefore, we can generalize our main conclusion to $r$ as the ES index: the quantitative impact of $p H$ over a wide range of credible conditions is also very small for Pearson's correlation, and is negligible in practical terms.

(d) The statistical model. We have made our calculations for simplicity under a fixed effect model, although psychologists rarely use it. The random effects model is generally considered more realistic (Borenstein, Hedges, Higgins, \& Rothstein, 2010). In order to show that our main result does not depend on the assumed model, we have performed a Monte Carlo simulation (see the syntax code in appendix S2 in the supplemental material). We have generated 100,000 samples for each condition. For the conditions, we have defined four mean ES values $(0,0.20,0.50$, and 0.80$)$ and three representative variance values $(0.05,0.10$, and 0.15$)$ (RubioAparicio et al., 2018). By crossing the 12 conditions with the same $p H$ levels as in the other sections, we have obtained the results of table S2. As can be seen, the levels of bias are still 
very low; in no case does the mean bias reach one hundredth.

(e) Broadening the operational definition of marginally significant. We have defined $p H$ as illegitimate transfers of results from the region in which $.05<p \leq .10$ to that of $.025<p \leq .05$. We believe this is a reasonable operationalization of $p H$, but one could ask how much the bias will change if we extend it, for example to the region of $.05<p \leq .20$ (i.e. $0.84 \leq T$ $<1.645)$. Table S3 shows the level of bias obtained with this new operational definition of $p H$. Beyond the modification in the operational definition of "marginally significant", the rest of the conditions are the same as in the calculations in Table 1

With such a broad definition of $p H$, we obtain again small levels of bias. The bias exceeds two hundredths only in a few conditions, especially with small samples $(\mathrm{N}=15)$ and high percentages of transferred studies $(q>.30)$. Only in one of the conditions does it reach three hundredths $(\delta=0.50$; $\mathrm{N}=15 ; q=.50)$.

(f) Variations in the sample sizes. To facilitate the calculations, we have assumed that the sample sizes of the studies are constant $(\mathrm{N}=15$ or $\mathrm{N}=30$ ), arguing that if they were variable, the results would not change significantly. In order to avoid any doubt in this regard (and following the suggestion of a reviewer) we have used a simulation methodology to recalculate the bias in such circumstances (see supplemental material). Specifically, we have generated 100,000 samples for each condition, associating to each one a random sample size, following the distribution proposed by Rubio-Aparicio et al., 2018). As expected, the results (Table S4) show that the size of the bias does not depend on whether the sample sizes vary. With variable sample sizes the bias is still very small.

(g) The meta-analytical strategy. The $p H$ has been studied through empirical distributions of ES estimates, probably already affected by $P B$. This has probably led to thinking of it more in terms of the combined effect of both factors than in the isolated effect of $p H$. When $P B$ exists and we ignore it, the combined estimate may suffer significant distortions (e.g., Carter, Schönbrodt, Gervais, \& Hilgard, 2019). An efficient strategy to avoid the effects of $P B$ is to analyze only significant studies, if a reasonable number of these are available (Simonsohn et al., 2014a; van Assen et al., 2015). It is assumed that $P B$ increases the frequency of studies with non-significant results that remain in the file-drawer but does not affect the number of significant ones. Therefore, we calculate also the effect that $p H$, as we have defined it, would have on combined estimates that are carried out under the strategy of including only significant studies, such as with the p-curve method.

We have used the function of the $p$-curve generated from a statistical test normally distributed (Ulrich \& Miller, 2018, equation 7), restricted to the range of significant $p$ values (left half of Figure 1, without $p H$ ). We have recalculated the density of each value under different probabilities of transferring a result with a $p$ value between .10 and .05 to the re- gion between .05 and .025 (again with values of $q$ from 0 to .50). With the new densities, we have fitted a curve with the same function. The values of the parameters thus obtained for different values of $q$ appear in Table S5. It is clear that the bias can be very large, especially when the effect is null or small. Figure 3 shows the effect of $p H$ when fitting a $p$ curve. The original function is that of the left segment drawn with a continuous line. The $p H$ is reflected in the increase in heights in the right segment. When we take the two segments and force a fit using a function like the original by least squares, the result is that of the figure drawn with a dashed line. This function appears above the original, before the effect of $p H$. As Simonsohn, Nelson and Simmons pointed out, the effect of $p H$ when using $p$-curve is an underestimation of the parametric effect size.

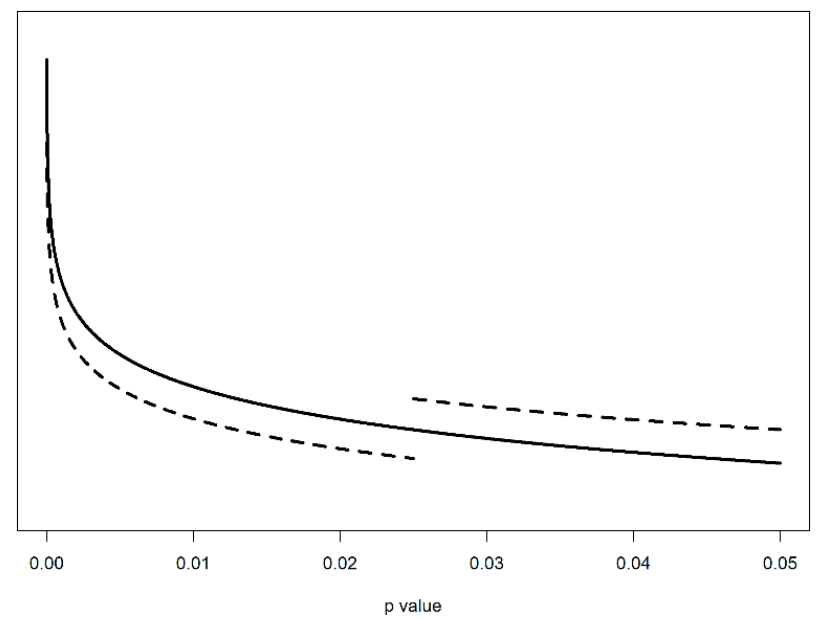

Figure 3. Effect of $p H$ when fitting a $p$-curve. The dashed line (distribution of $p$ values with $p H)$ is divided into two segments. The one on the left is not affected by $p H$; the one on the right is elevated due to the transfer of $p$ values $(p H)$. The solid line represents the fitted function that provides the $p$ curve from the two segments together.

In summary, in this section we have shown that by changing the analytic scenario in several ways, the size of the bias remains small, and in most conditions it is negligible. It only increases appreciably when we extend the operational definition of "marginally significant" to the threshold $p \leq$ .20. On the other hand, it is much larger when using $p$-curve with only significant studies. Our conclusion is that the quantitative impact of this type of $p H$ on the combined estimate of ES is generally small when the whole distribution is modeled. It is possible that in special circumstances the $p H$ affects the empirical distribution of the $E S$ values in a given body of studies more than in the scenarios analyzed here. However, in the vast majority of situations that we can realistically imagine, the impact is very small. To achieve a greater bias, we would have to assume much exaggerated conditions. For example, with $\delta=0.50$ and $\mathrm{N}=15$ even if the percentage of marginally significant studies that end up being significant as a result of $p H$ were $100 \%$, the bias would still be less than 0.02 . A bias of five hundredths, $|\mathrm{E}(d)-\delta|=$ 
0.05 , could be achieved, for example, if the marginally significant condition is extended to studies with $.05<p \leq .30$ (i.e., $0.52 \leq T<1.645)$ and it is assumed that $50 \%$ of these studies are converted to significant $p$-values through QRPs. These conditions involve a massive use of $Q R P s$, which we do not believe realistically represent their prevalence in psychology.

\section{Comparing the magnitude of the effects of $p H$ and $P B$}

Our main conclusion is that the magnitude of the effect of $p H$ is generally small. Of course, the label "small" is ambiguous; we have not defined operationally a priori what a "small" bias is. In most of the conditions analyzed, the bias is less than one hundredth. The indices of $E S$ analyzed, $d$ and $r$, are often reported to two decimal places. Then the smallest possible difference due to the effect of any factor is one hundredth (and a bias smaller than \pm .005 is negligible). That is why one hundredth is the unit of measure when evaluating our results. However, in order to assess the effects of $p H$ in relative terms we have made an additional analysis, comparing the quantitative effects of $p H$ with those of $P B$, the main threat in meta-analysis. We have calculated the quantitative impact on the combined estimation of the $E S$ when there is no $p H$, but there are different degrees of $P B$ and we ignore its effects. Table $\mathrm{S} 6$ shows the size of the bias when a proportion of non-significant studies is censored (they remain in the file drawer). For example, in the condition with $\delta=0.50$ the bias that occurs when half of the non-significant studies remain in the file drawer $(s=.50)$ is much greater than that produced by $p H$. This is a realistic imputation of the publication rate of non-significant studies. If in this scenario the probability that the study provides non-significant results is .607 with $N=15$, and, for example, $40 \%$ of them are censored, approximately one in every four studies carried out will remain in the file drawer (a very realistic situation; see, for example, the estimates of Franco et al., 2014). In this scenario, the expected value of $d$ is 0.575 . Comparing this level of bias with those of Table 1 for each condition, the conclusion is clear that the bias produced by $P B$ (as defined here) is much greater than that produced by $p H$ (as defined here).

\section{Some good reasons for preventing and fighting $p H$}

All our arguments lead to the conclusion that the magnitude of the impact of the type of $p H$ we have focusing here on a meta-analysis when calculating combined estimates in a wide range of realistic situations is very small, even negligible. Of course, it is much smaller than the impact of $P B$ of credible size. However, we do not want to convey the message that we can stop worrying about this type of $p H$. Quite the opposite. In the previous section, we have explained the reasons why we believe that it is not necessary to worry very much about its impact on the combined estimates of the ES obtained by the meta-analyst. Nevertheless, $p H$ has other consequences, especially when assessing the results of primary investigations by themselves. We present some good reasons why we should worry about and prevent $p H$ :

(a) The researcher crosses the line of scientific ethics, turning his or her work into a game in which opportunistic behaviors are put to work for personal gain in terms of career advancement. They reverse the priorities, putting personal profit ahead of the progress of knowledge acquired through a rigorous application of the scientific method (DeCoster et al., 2015). From a qualitative perspective, any prevalence of $Q R P s$ will be always too much. Furthermore, it is worrying that the presence of cues of QRPs is increasing, probably because the pressure to publish has been growing in the last two decades (De Winter \& Dodou, 2015; Holtfreter et al., 2019; Leggett et al., 2013).

(b) A falsely significant result may be confusing for other researchers, encouraging hypotheses without sufficient support that justifies following them with new research, thus wasting time and resources.

(c) The presence of QRPs reduces the confidence of researchers in previously published results. Science should be a cooperative task of honest collaboration among scientists. If this confidence is impaired, researchers feel the need to test the results of other researchers before accepting them as valid, wasting their time and investing additional resources unnecessarily.

(d) The same happens to professionals who could apply the advancements provided by scientists. They can reduce the amount of transfers to their professional practices if they do not fully trust the process that led to the conclusions (including results that are true and useful).

(e) If $Q R P s$ are present and the society knows it, then public confidence in the value of science is eroded, and faith in scientific contributions is tainted to the extent that public policy is less influenced by research into significant problems like global warming, environmental contamination, and species extinctions (Anvari \& Lakens, 2019).

(f) Confirmatory bias must be actively fought, rather than encouraged. Many young researchers normalize QRPs without being aware of being influenced by confirmatory bias. Deep inside the human mind rests the idea that our perception is more objective than that of others (Ross, 2018) and from there it easily progresses to the idea that our perceptions and intuitions are more credible than our own data. In the end, QRPs are cynically justified as acceptable behavior for the sake of scientific progress. The need to confirm previous beliefs, plus poor methodological and statistical training, within a social context in which "alternative truths" and "alternative facts" are accepted and normalized, can lead researchers to believe that what they do is correct.

(g) Incorrect conceptualizations of research methodology and statistics can be perpetuated. For example, by fostering the belief that the use of small samples makes research more efficient in terms of speed of publication, or attaching exag- 
gerated importance to the observation that $p<\alpha$. Researcher's ritualized behaviors can lead to incorrect interpretations, and the QRPs feedback those behaviors and pervasively reinforce their ritualization.

In summary, the arguments along this paper should not lead us to the conclusion that $p H$ is not a big problem for science and that we can therefore ignore it. It has serious consequences, especially at the level of primary studies. Those consequences justify implementing measures to prevent it (e.g., Botella \& Duran, 2019; De Boeck \& Jeon, 2018; Marusic, Wager, Utrobicic, Rothstein, \& Sambunjak, 2016; Sijtsma, 2016; Simmons, Nelson, \& Simonsohn, 2011). These resulting corrective proposals are promising. Extending their implementation will not only have a positive effect on scientific methodology alone. The mere fact that the society knows that the scientific community has been alerted to potential problems and is more diligent in improving methodological rigor will be an undoubted deterrent to reduce the prevalence of $Q R P$ s.

On the other hand, not all practices labeled as QRPs are always bad practices. Using a label with such a negative load can also be confusing. For example, performing interim analyses of the data collected so far and deciding whether to continue adding participants according to the result, is not a bad practice by itself and could be more efficient. It is only a bad practice if the researcher does not disclose what has been done, and if it is done outside of some regulated procedure. We know regulated ways to do partial analyses, so that the rate of type I errors is not inflated (e.g., Botella et al., 2006). Other types of $p H$, as analyzing the data in unexpected ways, is a source of discovery of unexpected patterns that enriches the process of science development (Wigboldus \& Dotsch, 2016). If we eliminate these unscheduled analyses from the practices of scientists, much of what their creativity can contribute is lost. What is a QRP is not to do these analyses, but not to report their exploratory nature.

\section{Limitations}

It can be argued that our way of modeling $p H$ is somewhat limited. Surely different $Q R P^{c}$ s have different effects. Sequential sampling and analysis (Botella et al., 2006) influences the $p$-curve in a different way to the parallel analysis of multiple dependent variables and the selection of the significant ones (Ulrich \& Miller, 2015), or to the application of various statistical techniques and reporting only the one that provides the most convenient value (e.g., Francis, 2012). Certainly, our way of modeling $p H$ is very close to the first of these $Q R P$ 's, which seems to be one of the most frequent in some fields of psychology. It would be necessary to study other scenarios focused on other $Q R P$ 's, or even scenarios in which several or all are present at different rates.

We have focused on the impact of $p H$ in the absence of $P B$. In other recent studies, the focus has been on the combined impact of both effects, especially on their interaction (e.g., Carter et al., 2019; Friese \& Frankenbach, 2019). We believe that the scenario studied by us is interesting by itself. The argument for their combined study would be that it is more realistic, since both problems, $p H$ and $P B$, are likely to be present in a particular field. Furthermore, it is sometimes argued that it is the presence of $P B$ that encourages $Q R P$ 's as a means of getting a study published. This being true, we believe there are other motivations for $p H$. Many authors feel a certain intellectual, and even emotional, commitment to certain explanatory positions and models. Personal involvement with theories can also push QRP's in fields where there is no noticeable $P B$, where a non-significant result would have been published anyway.

Furthermore, the fights against $p H$ and $P B$ are of a very different nature. We can implement mechanisms to achieve some control a priori of the studies, or plan massive replications, to avoid PB. We can also use subsequent analytical strategies that allow us to estimate and correct potential bias. However, QRP's are very difficult to control and their effects are difficult to correct. They often occur in the private domain of the researcher, or in his circle of trust. Our results at least show that if we can control and correct the $P B$, then we will not have to worry much about the quantitative impact of $p H$ on the meta-analytical estimation. Our main message is that in the absence of $P B$ the effect of $p H$ in the metaanalytical combined estimate is very small. Therefore, an efficient strategy to improve the scientific practices is to concentrate our efforts on $P B$. That said, we must not forget that the small magnitude of the bias we have calculated can become a much larger bias under more extreme (although less frequent and realistic) conditions.

We have assumed two instrumental assumptions in our calculations. None of them has an empirical basis. The alternative to the first assumption would be to assume that within studies with marginally significant results, the probability of being subjected to $p H$ is greater the lower the $p$-value. However, any non-uniform function would be arbitrary, and the ones we have tested have not produced very different results. Even so, it is pending to try with some different functions. The alternative to the second assumption would be to assume that the new values, after the transfer to the region of significant ones, are distributed in such a way that the conditioned expected value changes. Any specific function would be arbitrary and, again, the ones we have tested have not produced very different results. A systematic study of these alternative functions is pending. But we do not believe that reasonable deviations from these assumptions will significantly change the results.

\section{Conclusions}

QRPs are a threat to the fairness when implementing the scientific method in practice. We must fight them for several good reasons. However, our main conclusion is that among these reasons is not that they have a great impact on the meta-analytical estimation of ES. Our calculations lead to the 
conclusion that their real quantitative impact in a wide range of credible meta-analytical conditions is small.

Several consequences derive from this conclusion. First, researchers should not use the detection of $p H$ as a speculative argument to justify deviations from the expected results. When they find deviations from what they expect larger than $0.02-0.03$ in terms of $d$ or $r$, they will need alternative explanations to the mere speculation that the source of the deviation could be $p H$ without providing evidence that indeed it is.

A second consequence is that the selection models for the study of $P B$ do not need to include additional complications derived from this practice. Modeling $P B$ is a complex

\section{References}

Anvari, F., \& Lakens, D. (2019). The replicability crisis and public trust in psychological science. Comprehensive Results in Social Psychology, 1-21.

Baker, M. (2016). Is there a reproducibility crisis? A Nature survey lifts the lid on how researchers view the 'crisis' rocking science and what they think will help. Nature, 533(7604), 452-455.

Bakker, M., van Dijk, A,. \& Wicherts, J. M. (2012). The Rules of the Game Called Psychological Science. Perspectives on Psychological Science, 7, 543554.

Banks, G. C., Rogelberg, S. G., Woznyj, H. M., Landis, R. S., \& Rupp, D. E. (2016). Evidence on questionable research practices: The good, the bad, and the ugly. Journal of Business and Psychology, 31:323-338.

Bishop, D. V., \& Thompson, P. A. (2016). Problems in using p-curve analysis and text-mining to detect rate of $\mathrm{p}$-hacking and evidential value. PeerJ, 4, e1715.

Borenstein, M., Hedges, L. V., Higgins, J. P. T., \& Rothstein, H. R. (2010). A basic introduction to fixed-effects and random-effects models for meta-analysis. Research Synthesis Methods, 1, 97-111.

Bosco, F. A., Aguinis, H., Singh, K., Field, J. G., \& Pierce, C. A. (2015). Correlational effect size benchmarks. Journal of Applied Psychology, 100, 431-449.

Botella, J., \& Duran, J. I. (2019). A meta-analytical answer to the crisis of confidence of Psychology. Anales De Psicología/ Annals of Psychology, 35(2), 350-356.

Botella, J., Ximénez, M. C., Revuelta, J., \& Suero, M. (2006). Optimization of sample size in controlled experiments: the CLAST rule. Behavior Research Methods, Instruments \& Computers, 38(1), 65-76.

Brodeur, A., Lé, M., Sangnier, M., \& Zylberberg, Y. (2016). Star wars: The empirics strike back. American Economic Journal: Applied Economics, 8(1), $1-32$

Carter, E. C., Schönbrodt, F. D., Gervais, W. M., \& Hilgard, J. (2019). Correcting for bias in psychology: A comparison of meta-analytic methods. Advances in Methods and Practices in Psychological Science, 2(2), 115-144.

Cohen, J. (1988). Statistical power analysis for the behavioural sciences, $2^{\mathrm{a}}$ ed. New York: Academic Press.

De Boeck, P., \& Jeon, M. (2018). Perceived crisis and reforms: Issues, explanations, and remedies. Psychological Bulletin, 144(7), 757.

De Winter, J. C., \& Dodou, D. (2015). A surge of p-values between 0.041 and 0.049 in recent decades (but negative results are increasing rapidly too). PeerJ, 3, e733.

DeCoster, J., Sparks, E. A., Sparks, J. C., Sparks, G. G., \& Sparks, C. W. (2015). Opportunistic biases: Their origins, effects, and an integrated solution. American Psychologist, 70(6), 499.

Earp, B. D., \& Trafimow, D. (2015). Replication, falsification, and the crisis of confidence in social psychology. Frontiers in Psychology, 6, 621.

Fanelli, D. (2009). How many scientists fabricate and falsify research? A systematic review and meta-analysis of survey data. PloS one, 4(5), e5738.

Fiedler, K., \& Schwarz, N. (2016). Questionable research practices revisited. Social Psychological and Personality Science, 7(1), 45-52.

Francis, G. (2012). Publication bias and the failure of replication in experimental psychology. Psychonomic Bulletin \& Review, 19(6), 975-991. task that requires considering multiple sources of distortion in the distribution of ES values (Hedges \& Vevea, 2005; Rothstein, Sutton, \& Borenstein, 2005). Our results show that $p H$ is not in general a problem that we need to take into account in the development of the models, thus avoiding unnecessary additional complexities when modeling $P B$.

Authors note: Supplemental material for this article can be accessed at: https://osf.io/yc5tp/?view_only=906c8c4f86054efc87cf677c69a709be. This research was supported by the Ministerio de Economía y Competitividad of Spain (project reference: PSI201782490-P). Thanks are due to James F. Juola for reviewing and helping with the final polish of the manuscript.

Franco, A., Malhotra, N., \& Simonovits, G. (2014). Publication bias in the social sciences: Unlocking the file drawer. Science, 345(6203), 1502-1505.

Friese, M., \& Frankenbach, J. (2019). p-Hacking and publication bias interact to distort meta-analytic effect size estimates. Psychological Methods. Advance online publication. https://doi.org/10.1037/met0000246

Hall, J., \& Martin, B. R. (2019). Towards a taxonomy of research misconduct: The case of business school research. Research Policy, 48(2), 414 427.

Hartgerink, C. H. (2017). Reanalyzing Head et al. (2015): Investigating the robustness of widespread p-hacking. PeerJ, 5, e3068.

Head, M. L., Holman, L., Lanfear, R., Kahn, A. T., \& Jennions, M. D. (2015). The extent and consequences of p-hacking in science. PLoS Biology, 13(3), e1002106.

Hedges, L. V., \& Vevea, J. L. (2005). Selection method approaches. In H. R. Rothstein, A. J. Sutton, \& M. Borenstein (Eds.), Publication bias in metaanalysis: Prevention, assessment and adjustments ( $p$. 145-174). Chichester, England: John Wiley \& Sons.

Holtfreter, K., Reisig, M. D., Pratt, T. C., \& Mays, R. D. (2019). The perceived causes of research misconduct among faculty members in the natural, social, and applied sciences. Studies in Higher Education, 1-13.

Ioannidis, J. P., \& Trikalinos, T. A. (2007). An exploratory test for an excess of significant findings. Clinical Trials, 4(3), 245-253.

John, L. K., Loewenstein, G., \& Prelec, D. (2012). Measuring the prevalence of questionable research practices with incentives for truth telling. Psychological Science, 23(5), 524-532.

Johnson, N. L., Kotz, S., \& Balakrishnan, N. (1994). Continuous Univariat Distributions (2nd edition). New York, John Wiely Sons. Inc Vol. 2.

Kraemer, H. C., Gardner, C., Brooks, J., \& Yesavage, J. A. (1998). Advantages of excluding underpowered studies in meta-analysis: Inclusionist versus exclusionist viewpoints. Psychological Methods, 3(1), 23-31.

Krawczyk, M. (2015). The search for significance: a few peculiarities in the distribution of $\mathrm{P}$ values in experimental psychology literature. PloS One, 10(6), e0127872.

Lane, D. M., \& Dunlap, W. P. (1978). Estimating effect size: Bias resulting from the significance criterion in editorial decisions. British Journal of Mathematical \& Statistical Psychology, 31, 107-112.

Leggett, N. C., Thomas, N. A., Loetscher, T., \& Nicholls, M. E. (2013). The life of p: "Just significant" results are on the rise. The Quarterly Journal of Experimental Psychology, 66(12), 2303-2309.

Marszalek, J. M., Barber, C., Kohlhart, J., \& Cooper, B. H. (2011). Sample size in psychological research over the past 30 years. Perceptual and Motor Skills, 112(2), 331-348.

Martinson, B. C., Anderson, M. S., \& De Vries, R. (2005). Scientists behaving badly. Nature, 435 (7043), 737.

Marusic, A., Wager, E., Utrobicic, A., Rothstein, H. R., \& Sambunjak, D. (2016). Interventions to prevent misconduct and promote integrity in research and publication. Cochrane Database of Systematic Reviews, (4).

Mueller, G. P. (2018). When the search for truth fails: A computer simulation of the impact of the publication bias on the meta-analysis of scientific literature. Scientometrics, 117(3), 2061-2076. 
Pashler, H., \& Harris, C. R. (2012). Is the replicability crisis overblown? Three arguments examined. Perspectives on Psychological Science, 7(6), 531536.

Pashler, H., \& Wagenmakers, E.J. (2012). Editors' Introduction to the Special Section on Replicability in Psychological Science: A Crisis of Confidence? Perspectives on Psychological Science, 7, 528-530.

Richard, F. D., Bond, C. F., Jr., \& Stokes-Zoota, J. J. (2003). One hundred years of social psychology quantitatively described. Review of General Psychology, 7, 331-363.

Ross, L. (2018). From the fundamental attribution error to the truly fundamental attribution error and beyond: My research journey. Perspectives on Psychological Science, 13(6), 750-769.

Rothstein, H. R., Sutton, A. J., \& Borenstein, M. (Eds.) (2005). Publication bias in meta-analysis: Prevention, assessment, and adjustments. Nueva York: Wiley.

Rubio-Aparicio, M., Marín-Martínez, F., Sánchez-Meca, J., \& López-López, J. A. (2018). A methodological review of meta-analyses of the effectiveness of clinical psychology treatments. Behavior Research Methods, 50(5), 2057-2073

Schneck, A. (2018). Examining publication bias-a simulation-based evaluation of statistical tests on publication bias. PeerJ, 5, e4115.

Sijtsma, K. (2016). Playing with data-or how to discourage questionable research practices and stimulate researchers to do things right. Psychometrika, 81(1), 1-15.

Simmons, J. P., Nelson, L. D., \& Simonsohn, U. (2011). False-positive psychology: Undisclosed flexibility in data collection and analysis allows presenting anything as significant. Psychological Science, 22(11), 1359-1366.
Simonsohn, U., Nelson, L. D., \& Simmons, J. P. (2014a). P-curve: a key to the file-drawer. Journal of Experimental Psychology: General, 143(2), 534.

Simonsohn, U., Nelson, L. D., \& Simmons, J. P. (2014b). p-curve and effect size: Correcting for publication bias using only significant results. Perspectives on Psychological Science, 9, 666-681.

Stricker, J., \& Günther, A. (2019). Scientific misconduct in psychology: A systematic review of prevalence estimates and new empirical data. Zeitschrift für Psychologie, 227(1), 53.

Ulrich, R., \& Miller, J. (2015). p-hacking by post hoc selection with multiple opportunities: Detectability by skewness test?: Comment on Simonsohn, Nelson, and Simmons (2014). Journal of Experimental Psychology: General, 144(6), 1137-1145.

Ulrich, R., \& Miller, J. (2018). Some properties of p-curves, with an application to gradual publication bias. Psychological Methods, 23(3), 546.

van Aert, R. C., Wicherts, J. M., \& van Assen, M. A. (2019). Publication bias examined in meta-analyses from psychology and medicine: A metameta-analysis. PloS One, 14(4), e0215052.

van Assen, M. A. L. M., van Aert, R. C. M., \& Wicherts, J. M. (2015). Metaanalysis using effect size distributions of only statistically significant studies. Psychological Methods, 20, 293-309.

Wigboldus, D. H., \& Dotsch, R. (2016). Encourage plaving with data and discourage questionable reporting practices. Psychometrika, 81(1), 27-32.

Yong, E. (2012). In the wake of high-profile controversies, psychologists are facing up to problems with replication. Nature, 485, 298-300. 
anales de psicología / annals of psychology, 2021, vol. $37, \mathrm{n}^{\circ} 1$ (january) 